Objectives To examine the overall association between age and return-to-work (RTW), understand the extent to which functional, psychosocial, organizational, life-stage related factors indirectly explain these associations, and examine whether there is a remaining direct proportion not mediated by these factors.

Methods We used survey data from a prospective cohort of injured workers in Victoria, Australia. Participants were recruited during the 2014 to 2015 period from monthly samples of claimants identified by the compensation system. Path models examined the relationship between age and RTW, and the proportion mediated via functional, psychosocial, organizational, life-stage related factors.

Results Older age was associated with non-RTW, although the pattern was not observed consistently across follow-up surveys. A proportion of the overall relationship between age and nonRTW was explained by functional and life-stage factors and RTW status at previous time points.

Conclusion Findings underscore the importance of moving beyond age measured only in chronological years, towards more complex conceptual and analytical models that recognize age as a multidimensional construct.

\section{P-333 USING CANJEM TO EXAMINE THE ASSOCIATION BETWEEN OCCUPATIONAL EXPOSURE TO SELECTED METALS, METALLOIDS, AND WELDING FUMES AND BRAIN CANCER IN THE INTEROCC POOLED INTERNATIONAL CASE-CONTROL STUDY}

Romain Pasquet, Jack Siemiatycki, Lesley Richardson, Elisabeth Cardis, Anita Koushik
${ }^{1}$ Centre de recherche du Centre hospitalier de I'Université de Montréal (CRCHUM), Canada

\subsection{6/OEM-2021-EPI.273}

Introduction Exposure to metallic compounds may contribute to the etiology of brain cancer; however, few epidemiologic studies have examined this potential association.

Objective To examine occupational exposure to 21 metallic compounds in relation to the risk of glioma and meningioma.

Methods INTEROCC is an international consortium of seven brain cancer case-control studies using a common protocol. Among 1,917 glioma cases, 1,827 meningioma cases, and 5,475 controls in the pooled INTEROCC population, job histories were collected and transformed into histories of exposure to 21 metallic compounds by linkage to the Canadian job-exposure-matrix. Three metrics of exposure were calculated for each agent: ever exposed, duration of exposure, and cumulative exposure. Conditional logistic regression was used to estimate the odds ratios (ORs) and their 95\% confidence intervals (95\% CIs) for the association between the three metrics of exposure and both glioma and meningioma.

Results There was no evidence of associations between our selected agents and glioma. There were positive associations, with ORs ranging from 1.20 to 2.40 , between meningioma and several of the metallic compounds, most notably zinc compounds, lead fumes, chromium VI compounds, soldering fumes, metal oxide fumes, and soldering fumes. Overall, our results were similar to two previous studies based on INTEROCC that examined five of the metallic compounds included in this study, using a modified version of the Finish job-exposure-matrix.
Conclusion Our results are suggestive of positive associations between exposure to metallic compounds, particularly metallic fumes, and meningioma, but not glioma.

\section{P-334 OCCUPATIONAL EXPOSURES EXPERIENCED BY MONTRÉAL WOMEN PARTICIPATING IN TWO CASE- CONTROL STUDIES}

${ }^{1}$ Romain Pasquet, Emmanuelle Batisse, France Labrèche, Mark Goldberg, Vikki Ho. 'Centre de recherche du Centre hospitalier de I'Université de Montréal (CRCHUM), Canada

\subsection{6/OEM-2021-EPI.274}

Introduction Women constitute nearly half of the workforce. However, most of our knowledge about occupational diseases come from studies conducted on men.

Objective To describe occupational exposures experienced by women.

Methods Two case-control studies of postmenopausal breast cancer were conducted in Montreal in 1996 and in 2011. Questionnaires on lifetime occupational history were administered during in-person or telephone interviews. Experts reviewed subjects' work history, assessing exposure to a list of 258 chemicals. Chemicals that were deemed to be present were categorized by concentration ('low', 'medium', 'high'), where low represented a background occupational level and high was the highest level experienced in that work environment. We pooled exposure information from both studies by time period and by age of exposure.

Results In both studies combined, the three most prevalent exposures were cleaning agents, ozone, and organic solvents; the jobs in which these top 3 agents were present included nurses and waitresses (cleaning agents), secretaries and clerks (ozone), and housekeepers and elementary school teachers (organic solvents). For cleaning agents and ozone, most exposures occurred at a low concentration (>98\%) while slightly higher exposures to organic solvents were found (14\% medium and $2 \%$ high). The top 3 agents by time period were: <1950, fabric dust, aliphatic aldehydes, and cotton dust; 1950-1969, cleaning agents, aliphatic aldehydes, and organic solvents; 1970-1989, cleaning agents, ozone, and aliphatic alcohols; and $\geq 1990$, ozone, cleaning agents and aliphatic alcohols. The prevalence of exposures differed for women exposed earlier versus later in their working life (predominant agents $\leq 35$ years of age: cleaning agents, aliphatic aldehydes, aliphatic alcohols; $>35$ years of age were ozone, cleaning agents, organic solvents).

Conclusion Occupational exposures of women remain understudied; bringing out inconspicuous exposures can help better assess women's occupational risks.

\section{P-336 GENDER DIFFERENCES IN OCCUPATIONAL EXPOSURE IN THE CANADIAN JOB-EXPOSURE-MATRIX (CANJEM)}

${ }^{1}$ Romain Pasquet, Jérôme Lavoué, Jack Siemiatycki, Marie-Elise Parent, France Labrèche, Mark Goldberg, Vikki Ho. 'Centre de recherche du Centre hospitalier de I'Université de Montréal (CRCHUM), Canada

\subsection{6/OEM-2021-EPI.275}

Introduction One of the principal challenges in communitybased occupational studies is retrospective assessment of exposure. Job-exposure-matrices (JEMs) have been proposed as a 